\title{
Preoperative factors associated with adverse events during awake craniotomy: analysis of 609 consecutive cases
}

\author{
Hirokazu Takami, MD, PhD, Nikki Khoshnood, and Mark Bernstein, MD, MHSc, FRCSC \\ Division of Neurosurgery, Toronto Western Hospital, Toronto, Ontario, Canada
}

OBJECTIVE Awake surgery is becoming more standard and widely practiced for neurosurgical cases, including but not limited to brain tumors. The optimal selection of patients who can tolerate awake surgery remains a challenge. The authors performed an updated cohort study, with particular attention to preoperative clinical and imaging characteristics that may have an impact on the viability of awake craniotomy in individual patients.

METHODS The authors conducted a single-institution cohort study of 609 awake craniotomies performed in 562 patients. All craniotomies were performed by the same surgeon at Toronto Western Hospital during the period from 2006 to 2018. Analyses of preoperative clinical and imaging characteristics that may have an impact on the viability of awake craniotomy in individual patients were performed.

RESULTS Twenty-one patients were recorded as having experienced intraoperative adverse events necessitating deeper sedation, which made the surgery no longer "awake." In 2 of these patients, conversion to general anesthesia was performed. The adverse events included emotional intolerance of awake surgery $(n=13)$, air embolism $(n=3)$, generalized seizure $(n=4)$, and unexpected subarachnoid hemorrhage $(n=1)$. Preoperative cognitive decline, dysphasia, and low performance status, as indicated by the Karnofsky Performance Status (KPS) score, were significantly associated with emotional intolerance on univariate analysis. Only a preoperative KPS score $<70$ was significantly associated with this event on multivariate analysis $(p=0.0057)$. Compared with patients who did not experience intraoperative adverse events, patients who did were more likely to undergo inpatient admission ( $p=0.0004$ for all cases; $p=0.0036$ for cases originally planned as day surgery), longer hospital stay $(p<0.0001)$, and discharge to a location other than home $(p=0.032)$.

CONCLUSIONS Preoperative physical status was found to be the most decisive factor in predicting whether patients can tolerate an awake craniotomy without complications, whereas older age and history of psychiatric treatment were not necessarily associated with adverse events. Patients who had intraoperative adverse events often had reduced chances of same-day discharge and discharge to home. Preoperative careful selection of patients who are most likely to tolerate the procedure is the key to success for awake surgery.

https://thejns.org/doi/abs/10.3171/2020.4.JNS20378

KEYWORDS awake surgery; glioma; intraoperative seizure; metastasis; surgical technique

A WAKE craniotomy is becoming more standardized, not just for brain tumor surgeries, but also for surgeries performed for conditions with other etiologies. ${ }^{1,2}$ Methodologies for awake surgeries have developed over time, making this surgery option more feasible and safer for patients. In brain tumor surgery for which the lesions are in or adjacent to critical structures such as areas crucial to motor, sensory, language, or cognitive functions, awake surgery is performed for the purpose of preserving these functions by using intraoperative stimulation in and around the lesions and monitoring the resulting symp- toms through interactions with the awake patients. ${ }^{3-7}$ In addition, awake surgeries are performed to expedite the patient's postoperative recovery by avoiding general anesthesia and intubation. ${ }^{2}$ This type of awake surgery is often used in "day surgery" or "outpatient surgery." At our center, patients who are sent to the day surgery unit are monitored for several hours after completion of the surgery, undergo postoperative scanning, and then, if the patients are neurologically and clinically stable, in most cases they are discharged to home. ${ }^{8-12}$

Although awake craniotomy has been recognized in

ABBREVIATIONS KPS = Karnofsky Performance Status; PCNSL = primary central nervous system lymphoma.

SUBMITTED February 6, 2020. ACCEPTED April 1, 2020.

INCLUDE WHEN CITING Published online June 26, 2020; DOI: 10.3171/2020.4.JNS20378. 
recent years as a useful surgical method in daily neurosurgical practice, this method is not achievable without the cooperation of other healthcare professionals, including anesthesiologists, nurses, and speech and occupational therapists. But above all, the cooperation of the patients themselves is essential. Patients planning to undergo awake craniotomy must understand the merits of awake versus asleep surgery and often must cooperate with the surgical team and be engaged in multiple tasks during the surgery to be assessed for their neurological functions., ${ }^{413}$ In contrast to asleep surgeries, during awake surgeries patients may have to tolerate some discomfort related to their body positions and the pain and discomfort caused by pinning to immobilize the head, incisions, airway protection, anxiety, nausea, etc..$^{14}$ Older age may be regarded as one of the negative indications for the ability to tolerate awake surgery, although surgeries for high-grade glioma and metastatic brain lesions, which are among the surgeries for which outcomes are most likely to be enhanced if performed on awake patients, are often performed in elderly patients. ${ }^{15,16}$ Appropriate selection of patients is not always feasible, and selection performed on a case-by-case basis seems to be the standard approach..$^{2,14}$

Here, we report a review of 609 cases of awake craniotomies performed in 562 separate patients, including 21 patients who experienced intraoperative adverse events, to investigate the preoperative factors that may be predictive of outcomes of these awake procedures.

\section{Methods}

\section{Data Source and Acquisition}

A comprehensive, prospectively maintained neurosurgical patient registry was retrospectively queried for cases of awake surgery performed by the senior author (M.B.) during the period from July 2006 to December 2018. Captured clinical endpoints included patient age and sex, presenting symptoms, lesion location and size, histopathological diagnoses, surgical details, intraoperative monitoring, intraoperative symptoms and events, postoperative symptoms, and discharge locations. Cognitive decline was evaluated by medical practitioners (neurosurgeon and anesthetists) who performed patient assessments by examining the patient and collecting information from family members at the time of visits to the outpatient clinic or preoperative care unit. All final histopathological diagnoses were made or confirmed at our institution. All pertinent components of the study were approved by our Institutional Review Board, including provision of an informed consent waiver for what was deemed a minimal-risk retrospective review.

\section{Awake Surgery Criteria, Surgical Procedures, and Outpatient Surgery}

As described in a previous report, ${ }^{17}$ our institution performs awake surgeries not only in patients with intraaxial lesions adjacent to critical functional structures, but also in patients for whom avoiding general anesthesia is expected to expedite recovery from surgery.

In these awake surgeries, light sedation was administered together with local anesthesia to the surgical incision site and to the pin fixation sites. Circumferential block anesthesia was used for the incision site. Image-guided frameless navigation was also employed for all cases. In patients in whom lesions were found on imaging to be close to important functional structures, intraoperative electrical stimulation mapping with a bipolar stimulator was used to identify the functional cortical and subcortical areas. Preoperative administration of antiseizure medication was not routinely performed unless the patient was already taking it. Postoperatively, patients stayed in the postanesthesia care unit for observation for 2 to 4 hours before being discharged to the day surgery unit or an inhospital unit, based on the results of postoperative imaging and neurological status assessments. The indications for same-day discharge (outpatient surgery) have been outlined in detail in previous publications. ${ }^{8}$ Briefly, patients were selected for same-day discharge in collaboration with the anesthesiologists, who assessed the patients preoperatively for cases with a supratentorial tumor, availability of a caregiver, and immediate postoperative discharge to a location geographically close to the hospital.

Occasionally patients experienced discomfort during awake surgery and engaged in behavior characterized by rambunctiousness, restlessness, and agitation (collectively classified as emotional intolerance), which made keeping the patient awake and cooperative difficult and/or risky, so that the operator decided to abort the monitoring of awake neurological status during the procedure and converted mild sedation to deeper sedation.

\section{Statistical Analysis}

Nonparametric values were compared using the Wilcoxon test. Fisher's exact test was used for paired categorical data, and multivariate modeling was performed via Cox regression analysis. All statistical analyses were carried out using JMP 14 (SAS Institute Inc.). An alpha level of 0.05 was used to define statistical significance.

\section{Results}

\section{Patient Demographics}

This study included 609 surgeries (on average, 1 awake craniotomy every week for the 12-year study epoch) performed in 562 patients. The patient age range was 18 to 94 years, with a median age of 57 years and an average age of $55.9 \pm 15.7$ years. The female to male case ratio was 279 to 328 (Fig. 1A) for 254 female and 308 male patients.

Of the 562 study patients, 521 patients underwent 1 awake craniotomy, 38 patients underwent 2 awake craniotomies, 1 patient underwent 3, 1 patient underwent 4 , and 1 patient underwent 5 awake craniotomies. In 2 patients, 2 awake craniotomies were performed in a single session on the same day for bilateral lesions. In all other patients who had 2 or more craniotomies, the procedures were performed on different days. Each of the 609 total awake craniotomies was included as a separate case in the data analyses. In addition, because of the heterogeneity of the population of patients who underwent awake surgery during the 12-year study period, all of the data investigated were not available for all of the patients. 

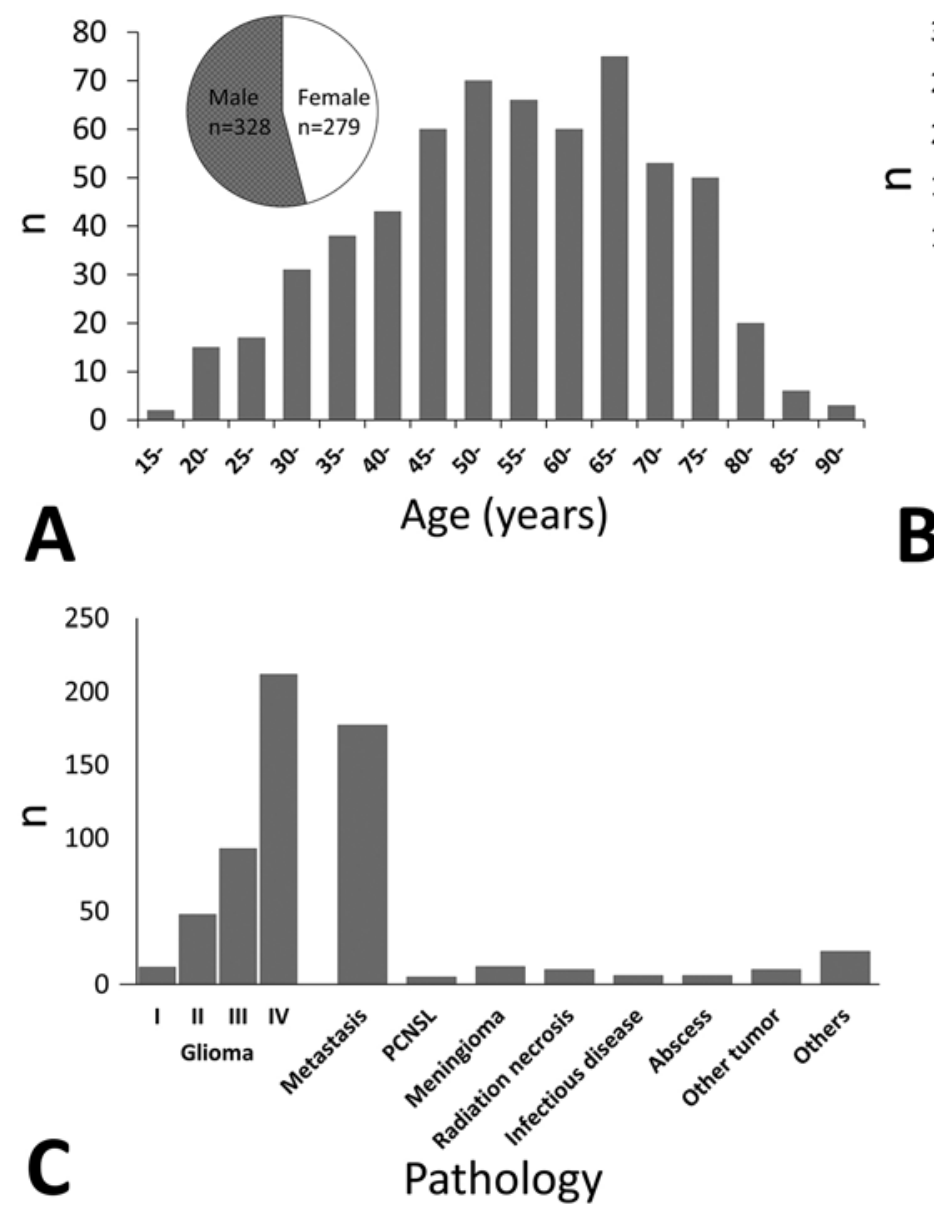

\section{Tumor Location, Imaging Findings, and Pathological Diagnosis}

The locations of the 609 resected lesions in the brain were as follows (Fig. 1B), according to area of the brain: right 263 , left 342 , bilateral 2 , and midline 2 ; and anatomical location: frontal lobe 285 , parietal 119 , temporal 112 , occipital 15 , fronto-temporal 2 , fronto-parietal 14 , temporo-occipital 1, temporo-parietal 6, parieto-occipital 3 , insula 16 , corpus callosum 10 , thalamus 20 , and other locations 6.

Histopathological diagnoses included glioma for 365 lesions (12 grade I, 48 grade II, 93 grade III, and 212 grade IV); metastasis, 173; radiation necrosis, 10; meningioma, 12; primary central nervous system lymphoma (PCNSL), 5 ; other tumor types, 10; infectious disease (such as toxoplasmosis, tuberculosis, and parasites), 6; abscess, 6; and other diseases, 22 lesions. Malignancy was defined as grade III/IV glioma, metastasis, and PCNSL (Fig. 1C).

\section{Pre- and Postoperative Symptomatology}

Before surgery, preoperative symptomatology was recorded for 594 patients, of whom 208 did not have symptoms and the remaining patients reported symptoms as follows: weakness in 214 patients (36.0\%), sensory deficits of the extremities in 85 (14.3\%), dysphasia/aphasia in 128 (21.5\%), visual field deficits in 38 (6.4\%), and cognitive

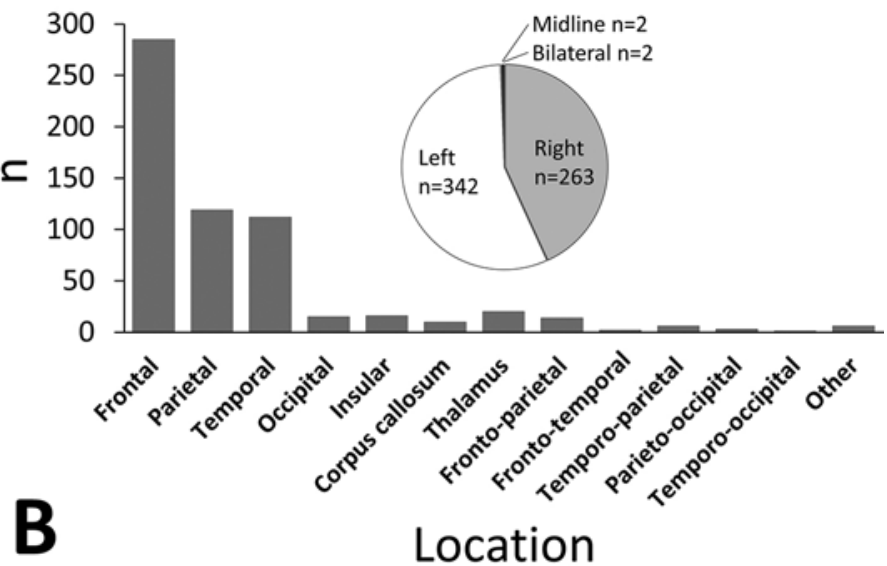

FIG. 1. A: Patient age distribution in awake craniotomy cases. The median age was 57 years (average $55.9 \pm 15.7$ years). The pie chart shows the female to male case ratio of 279 to 328 , for 254 female and 308 male patients. B: The locations of lesions targeted in the 609 cases of awake surgeries are shown. About half of the lesions were located in the frontal lobe $(46.9 \%, 285$ cases), followed by parietal (19.5\%, 119 cases) and temporal (18.4\%, 112 cases) lesions. The pie chart shows the side of the lesions, including 2 bilateral lesions (1 on each side, in a total of 2 patients) and 2 midline lesions. Left lesions were dominant (56.2\%, 342 lesions). C: Histopathological diagnoses or etiologies of the diseases that were treated in awake surgeries are shown. Gliomas were the most frequent, making up more than half of the lesions (59.9\%, 365 cases), followed by metastatic tumors $(28.4 \%, 173$ cases $)$.

decline in 125 patients (21.0\%). In 13 patients, information was lacking on preoperative symptoms. There were 57 patients $(10.5 \%)$ who had psychiatric illness before the surgery. Regarding performance status, there were 451 patients $(78.0 \%)$ who were independent in daily life (Karnofsky Performance Status [KPS] score $\geq 70$ ) of 578 patients for whom records were available (Fig. 2A-C).

\section{Adverse Events During Awake Surgery}

Adverse events occurred during awake surgery in 21 patients. The details of the preoperative, intraoperative, and postoperative clinical and radiological findings in these patients are summarized in Supplementary Table 1. In no case was the surgery discontinued-the intended goal of the surgery was always achieved. Adverse events included emotional intolerance in 13 patients; air embolism in 3 patients, which occurred in 1 patient with left parietal metastasis from lung adenocarcinoma (in a sitting position during surgery), 1 patient with a left frontal anaplastic oligodendroglioma (in a supine position), and 1 patient with a right parietal gliosarcoma (in a lateral position); generalized seizure in 4 patients; and an unexpected subarachnoid hemorrhage in 1 patient with a grade IV left frontal glioma (glioblastoma).

Among these events, in 2 cases ( 2 patients) the occurrence of air embolisms resulted in procedural and anes- 


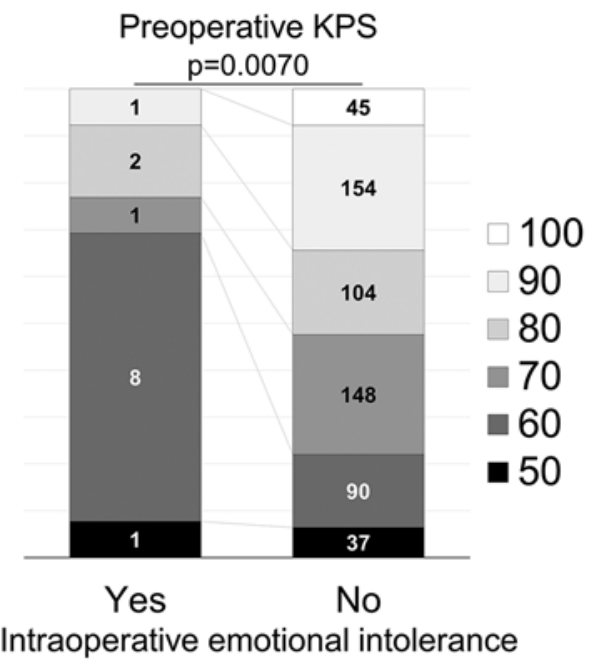

A Intraoperative emotional intolerance

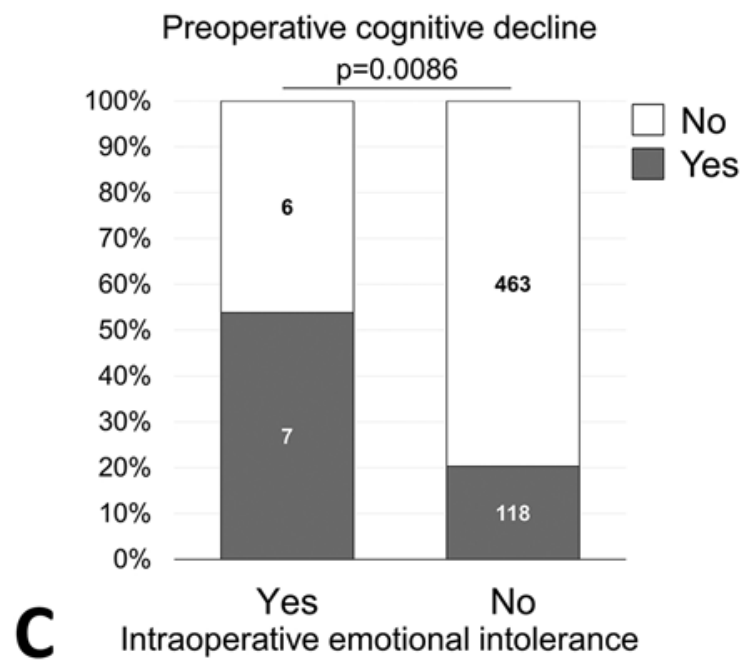

thesia changes from awake surgeries to surgeries with general anesthesia and airway management. Anesthesiologists performed endotracheal intubation in one of these patients (case 2 in Supplementary Table 1) and placed a laryngeal mask in the other patient (case 8 in Supplementary Table 1) and administered intravenous anesthetic and/ or inhalation anesthetic. Brain mapping was discontinued in both patients. Arterial lines were also placed to monitor hemodynamic stability, but this is not routinely used for awake craniotomies.

\section{Investigations of Intraoperative Emotional Intolerance of Awake Surgery}

There were 13 cases in which patients showed emotional intolerance of awake surgery that necessitated deeper sedation. Comparisons were performed of preoperative clinical and imaging factors to clarify those that may have contributed to intraoperative issues.

Tables 1 and 2 show the clinical and imaging factors that were assessed and the results of the univariate and multivariate statistical analyses. There were no statistically significant differences associated with patient age

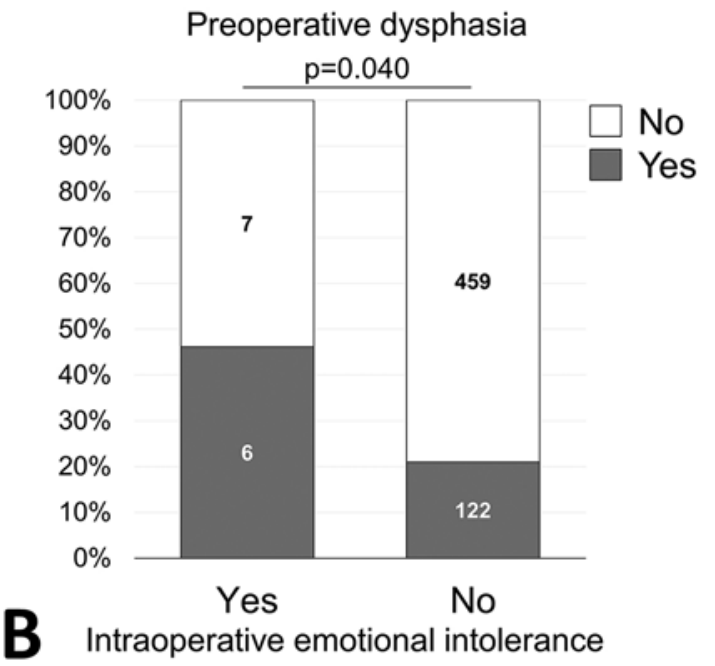

FIG. 2. A: Comparison of preoperative KPS scores in patients with intraoperative emotional intolerance $(n=13)$ and those without $(n=578)$. The scores were significantly different $(p=0.0070)$, especially for the ratios of the cases of independence in daily life (KPS $\geq 70,4$ of 13 vs 455 of 585 cases; $p=0.0005$ ). B: The rate of the presence of preoperative dysphasia was compared between the cases with intraoperative emotional intolerance $(n=13)$ and those without $(n=581)$, which revealed the deficit was higher in the prior group $(46.2 \%$ vs $21.0 \%, p=0.040)$. C: The rate of preoperative cognitive decline was compared between the cases with intraoperative emotional intolerance $(n=13)$ and those without $(n=581)$, which revealed the deficit was higher in the prior group ( $53.8 \%$ vs $20.3 \%, p=0.0086)$.

or sex, duration of surgery, first time versus redo surgery, left- versus right-sided lesion location, bilateral versus unilateral lesion, tumor volume, or presence versus absence of imaging enhancement, edema around lesions, malignancy, preoperative weakness, sensory deficit, visual field deficit, previous radiation therapy or chemotherapy, or intraoperative electrical stimulation.

On univariate analysis (Table 1), preoperative language deficit, preoperative cognitive decline, and low preoperative KPS score $(<70)$ were significantly associated with intraoperative emotional intolerance $(\mathrm{p}=0.040,0.0086$, and 0.0005 , respectively). Using these 3 factors as covariates, multivariate analysis revealed that preoperative KPS was the only statistically significant factor affecting intraoperative behavior $(\mathrm{p}=0.0057$, Table 2$)$.

\section{Outcomes Following Awake Surgery}

Among the 608 cases with available data, 377 cases were planned as day surgery and the other 231 cases were planned as surgery followed by inpatient admission (information was missing for 1 case). Of the 377 cases with planned day surgery, in 49 cases (13.0\%) patients were 
TABLE 1. Univariate analysis of clinical and imaging factors and emotional intolerance during awake surgery

\begin{tabular}{|c|c|c|c|c|}
\hline \multirow[b]{2}{*}{ Parameter } & \multirow[b]{2}{*}{ All } & \multicolumn{2}{|c|}{ Intraop Emotional Intolerance } & \multirow[b]{2}{*}{ p Value } \\
\hline & & Yes $(n=13)$ & No $(n=585)$ & \\
\hline Age, yrs & $56.1 \pm 15.6$ & $61.2 \pm 14.2$ & $56.0 \pm 15.7$ & 0.24 \\
\hline \multicolumn{5}{|l|}{ Sex } \\
\hline Female & 277 & 4 & 273 & 0.40 \\
\hline Male & 321 & 9 & 312 & \\
\hline \multicolumn{5}{|l|}{ First or redo op } \\
\hline First & 491 & 10 & 481 & 0.71 \\
\hline Redo & 106 & 3 & 103 & \\
\hline \multicolumn{5}{|l|}{ Intra- or extraaxial lesion } \\
\hline Intraaxial & 581 & 13 & 568 & 1.00 \\
\hline Extraaxial & 16 & 0 & 16 & \\
\hline \multicolumn{5}{|l|}{ Position } \\
\hline Left side & 338 & 8 & 330 & 1.00 \\
\hline Right side & 256 & 5 & 251 & \\
\hline Midline & 2 & 0 & 2 & \\
\hline Bilateral & 2 & 0 & 2 & \\
\hline \multicolumn{5}{|l|}{ Bilateral lesions $†$} \\
\hline Yes & 82 & 1 & 81 & 0.52 \\
\hline No & 516 & 12 & 504 & \\
\hline Tumor vol, $\mathrm{cm}^{3}$ & $29.6 \pm 30.4$ & $32.6 \pm 29.3$ & $29.5 \pm 30.4$ & 0.71 \\
\hline \multicolumn{5}{|l|}{ Lesion enhancement } \\
\hline Yes & 455 & 13 & 442 & 0.080 \\
\hline No & 130 & 0 & 130 & \\
\hline Brain edema around lesion & & 12 & & \\
\hline Yes & 436 & 11 & 425 & 0.31 \\
\hline No & 135 & 1 & 134 & \\
\hline \multicolumn{5}{|l|}{ Malignancy } \\
\hline Yes & 479 & 13 & 466 & 0.082 \\
\hline No & 119 & 0 & 119 & \\
\hline \multicolumn{5}{|l|}{ Preop weakness } \\
\hline Yes & 214 & 3 & 211 & 0.39 \\
\hline No & 380 & 10 & 370 & \\
\hline \multicolumn{5}{|l|}{ Preop sensory deficit } \\
\hline Yes & 85 & 0 & 85 & 0.23 \\
\hline No & 509 & 13 & 496 & \\
\hline \multicolumn{5}{|l|}{ Preop aphasia/dysphasia } \\
\hline Yes & 128 & 6 & 122 & $0.040^{*}$ \\
\hline No & 466 & 7 & 459 & \\
\hline \multicolumn{5}{|l|}{ Preop visual field deficit } \\
\hline Yes & 38 & 1 & 37 & 0.58 \\
\hline No & 556 & 12 & 544 & \\
\hline \multicolumn{5}{|l|}{ Preop cognitive decline } \\
\hline Yes & 125 & 7 & 118 & $0.0086^{*}$ \\
\hline No & 469 & 6 & 463 & \\
\hline \multicolumn{5}{|l|}{ Preop psychiatric issue } \\
\hline Yes & 57 & 2 & 55 & 0.32 \\
\hline No & 485 & 9 & 476 & \\
\hline
\end{tabular}


TABLE 1. Univariate analysis of clinical and imaging factors and emotional intolerance during awake surgery

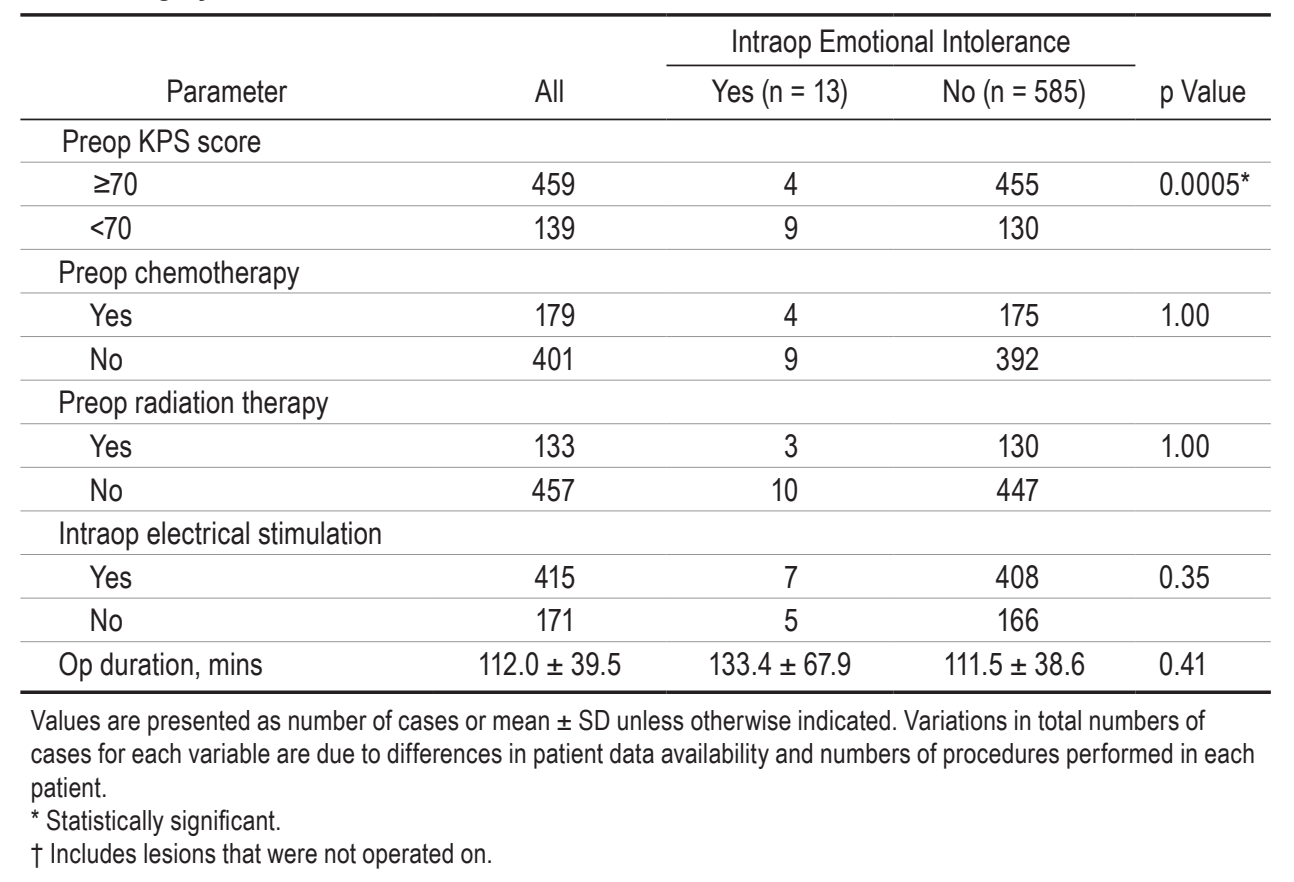

admitted from the day surgery unit to the inpatient ward for continuing care. The hospital stay was counted as the number of days including the day of surgery. Among 593 cases that included these data, in 521 cases the patients were discharged home, including 321 cases of day surgery, and in 72 cases patients were discharged to destinations other than home, including rehabilitation facilities and skilled nursing facilities.

In 21 cases patients experienced intraoperative adverse events. One case (case 11 in Supplementary Table 1) was omitted from the analysis due to the lack of information about "planned day surgery," "admission," "unplanned admission," and "hospital stay." Twenty cases were compared with the cases of patients who did not experience intraoperative adverse events to assess the variability of postoperative recovery among these groups (Table 3). The inpatient admission rate was higher for the group who experienced intraoperative adverse events, with $85.0 \%$ being admitted (17 of 20 cases), compared with $44.9 \%$ without adverse events (259 of 577 cases, $p=0.0004$; Fig. 3A). The variability in admission rates as a function of intraoperative adverse events was also found when looking at unplanned admission (i.e., patients who were supposed to be discharged on the same day of surgery but were admitted

TABLE 2. Multivariate analysis of clinical and imaging factors and intraoperative emotional intolerance

\begin{tabular}{|c|c|c|c|c|c|}
\hline \multirow[b]{2}{*}{ Variable } & \multirow[b]{2}{*}{ All } & \multicolumn{2}{|c|}{ Intraop Emotional Intolerance } & \multirow[b]{2}{*}{$\mathrm{HR}(95 \% \mathrm{Cl})$} & \multirow[b]{2}{*}{$\mathrm{p}$ Value } \\
\hline & & Yes $(n=13)$ & No $(n=585)$ & & \\
\hline \multicolumn{6}{|c|}{ Preop aphasia/dysphasia } \\
\hline Yes & 128 & 6 & 122 & $2.74(0.88-8.49)$ & 0.090 \\
\hline No & 466 & 7 & 459 & 1 & \\
\hline \multicolumn{6}{|c|}{ Preop cognitive decline } \\
\hline Yes & 125 & 7 & 118 & $2.31(0.71-7.58)$ & 0.17 \\
\hline No & 469 & 6 & 463 & 1 & \\
\hline \multicolumn{6}{|l|}{ Preop KPS score } \\
\hline$\geq 70$ & 451 & 4 & 455 & $5.54(1.56-19.65)$ & $0.0057^{*}$ \\
\hline$<70$ & 127 & 9 & 130 & 1 & \\
\hline
\end{tabular}

Values are presented as number of cases unless otherwise indicated. Variations in total numbers of cases for each variable are due to differences in patient data availability and numbers of procedures performed in each patient.

* Statistically significant. 
TABLE 3. Postoperative clinical course in cases with or without intraoperative adverse events

\begin{tabular}{|c|c|c|c|c|c|c|c|}
\hline \multirow[b]{2}{*}{ Parameter } & \multirow[b]{2}{*}{ All } & \multicolumn{2}{|c|}{ Adverse Events } & \multirow[b]{2}{*}{ p Value } & \multicolumn{2}{|c|}{ Emotional Intolerance } & \multirow[b]{2}{*}{$p$ Value } \\
\hline & & Yes $(n=21)$ & No $(n=577)$ & & Yes $(n=13)$ & No $(n=585)$ & \\
\hline \multicolumn{8}{|l|}{ Admission } \\
\hline Yes & 276 & 17 & 259 & $0.0004^{*}$ & 9 & 267 & 0.075 \\
\hline No & 321 & 3 & 318 & & 3 & 318 & \\
\hline Unknown & 1 & 1 & 0 & & 1 & 0 & \\
\hline \multicolumn{8}{|c|}{ Unplanned admission } \\
\hline Yes & 49 & 6 & 43 & 0.092 & 3 & 46 & 0.11 \\
\hline No & 227 & 11 & 216 & & 6 & 221 & \\
\hline Hospital stay, days & $3.0 \pm 5.5$ & $5.2 \pm 4.3$ & $3.0 \pm 5.5$ & $<0.0001^{*}$ & $4.5 \pm 3.8$ & $3.0 \pm 5.5$ & $0.0085^{*}$ \\
\hline \multicolumn{8}{|l|}{ Discharge to home } \\
\hline Yes & 521 & 15 & 506 & $0.032^{*}$ & 9 & 512 & 0.061 \\
\hline No & 72 & 6 & 66 & & 4 & 68 & \\
\hline Unknown & 5 & 0 & 5 & & 0 & 5 & \\
\hline
\end{tabular}

Values are presented as number of cases or mean \pm SD unless otherwise indicated.

* Statistically significant.

as inpatients instead), and this was seen for $35.3 \%$ (6 of 17 cases) compared with $16.6 \%$ (43 of 259 cases, $\mathrm{p}=0.092$ ). Longer hospital stays were observed among patients with intraoperative adverse events, with an average hospital stay of 5.2 days compared with 3.0 days in unaffected patients ( $p<0.0001$; Fig. 3B). Another statistically significant difference observed was that discharge to locations other than the patient's home was more likely if the patient experienced an intraoperative adverse event $(28.6 \%$ [ 6 of 21 cases] compared with $11.5 \%$ [66 of 572 cases], $\mathrm{p}=$ 0.032; Fig. 3C).

This tendency was also observed in the data for patients who experienced intraoperative emotional intolerance. The inpatient admission rate was higher in patients with emotional intolerance ( 9 of 12 cases, $75.0 \%$ ) than in those without (267 of 585 cases, 45.6\%; $\mathrm{p}=0.075$ ), especially the unplanned admission rate (3 of 9, 33.3\%, vs 46 of 267 cases, $17.2 \% ; \mathrm{p}=0.11)$, the hospital stay was longer (4.5 vs 3.0 days, $\mathrm{p}=0.0085)$, and the discharge location was more likely not to be home (4 of 13 patients, $30.8 \%$, vs 68 of $580,11.7 \% ; \mathrm{p}=0.061)$, although these differences were statistically marginal.

\section{Discussion}

We present what is to our knowledge a unique analysis on "failed" cases of awake craniotomy surgeries with respect to the contributing factors as demonstrated in the available imaging and clinical data. Cumulatively, 609 cases were retrospectively reviewed (data were recorded prospectively) in an analysis that revealed that language deficit, cognitive decline, and low performance status before the surgery were significantly associated with intraoperative emotional intolerance on univariate analysis, and notably low preoperative performance status was the only statistically significant indicator.

Intraoperative seizure and lack of cooperation are regarded as two major neurosurgical complications during awake craniotomy. ${ }^{1}$ In the current study, air embolism, which is also a recognized complication in awake craniotomy, ${ }^{18,19}$ was encountered in 3 cases $(0.49 \%)$. Interestingly, we found that older patient age was unrelated to intraoperative emotional intolerance. Even after including all of the intraoperative adverse events $(n=21)$, age was proven to be unrelated (data not shown). In the current series, there were 29 patients aged 80 years and older, including 3 patients aged 90 years and older, and none of these patients encountered significant intraoperative adverse events. This finding suggests that older age is not a contraindication for awake surgery, corroborating a previous report, ${ }^{16}$ as long as the patients have fair preoperative performance status, namely a KPS score $\geq 70$. Furthermore, preoperative psychological illness was also not associated with adverse behavior during awake surgery.

The patient performance status, as indicated by the KPS score in this study, can be impacted by any type of neurological deterioration, including motor, language, and cognitive deficits. In the present study the latter two were associated with intraoperative emotional intolerance on univariate analysis. Whereas most of the patients with intraoperative emotional intolerance harbored dysphasia or cognitive decline (11 of 13 patients), motor deficit before surgery was identified in only 1 patient. There were 29 patients with a low KPS score $(<70)$, which was recorded as reflecting motor deficit only, and all but one of these 29 patients had a successful awake procedure. This finding suggests that not all patients with low KPS scores are at risk for adverse events during awake surgery, but weighing the risk factors, especially dysphasia and cognitive decline, may be indicated to appropriately assess the safety of awake surgery preoperatively.

The patients with intraoperative adverse events, including emotional intolerance during awake surgery, were shown to be more frequently admitted as inpatients, have longer hospital stays, and be less likely to be discharged to home than the patients who underwent the awake surgeries smoothly. This finding again highlights the point that preoperative performance status is important with regard to 

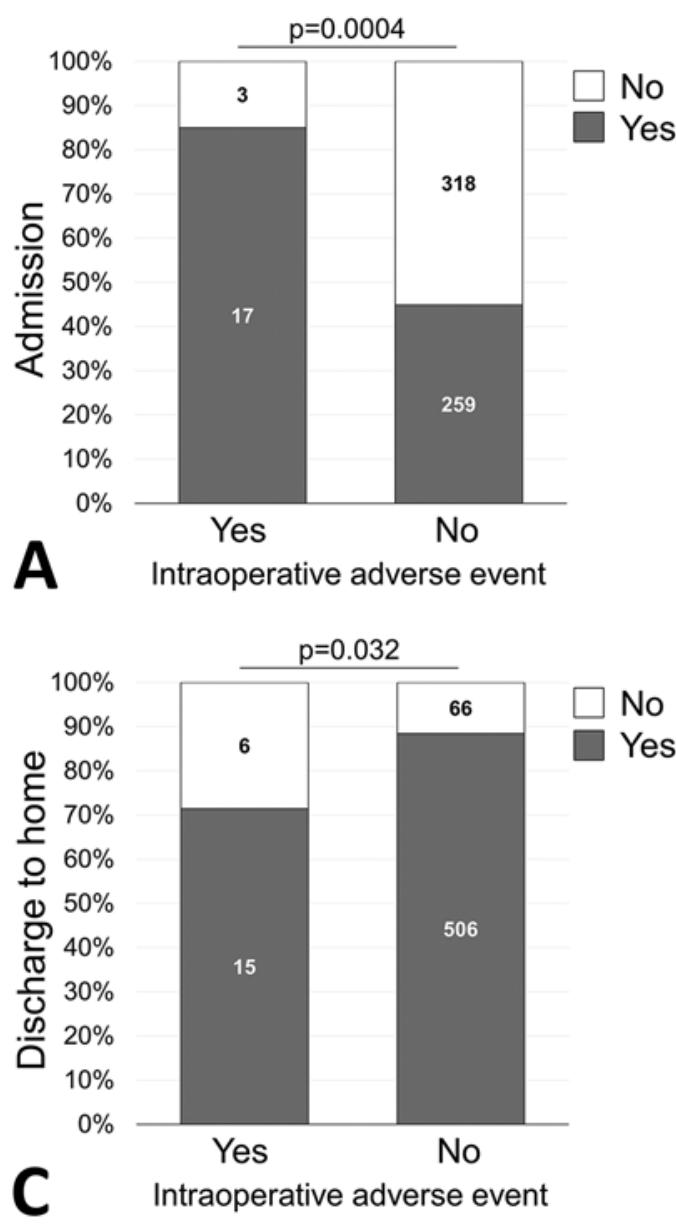

predicting patient postoperative status after awake craniotomy. The results from the current study are in line with those of a previous report that preoperative performance status was one of the predictors for early discharge after awake craniotomy in an analysis based on a smaller number of cases. ${ }^{11}$

Patients with brain tumors often have inadequate mental capacity, ${ }^{20}$ and assessment and evaluation of this factor is considered to be important before these patients undergo surgery. ${ }^{21}$ The results of this study corroborate this notion that sufficient preoperative evaluation of the patient clinical status is imperative for awake surgeries, and this information should be carefully integrated into the decision-making process as to whether patients can safely undergo while awake surgery or if they may benefit more from surgery while under general anesthesia. As long as the cases are appropriately selected, patient satisfaction with the procedure is known to be high., ${ }^{1,13}$

\section{Study Limitations}

There were several limitations in this study. First, the number of cases with intraoperative adverse events in the study cohort was small $(\mathrm{n}=21,3.4 \%)$, which made it challenging to assess preoperative factors for each type of adverse event (i.e., emotional intolerance, air embolism,

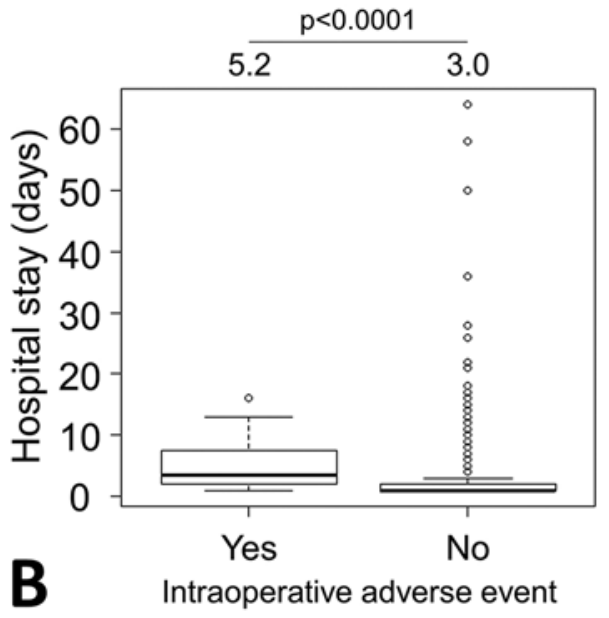

FIG. 3. A: The postoperative hospital admission rate was compared between the cases with intraoperative adverse events $(n=21)$ and those without $(n=577)$, which revealed that the rate was higher in the prior group ( $85.0 \%$ vs $44.9 \%, p=0.0004)$. B: The length of hospital stays after surgery was compared between the cases with intraoperative adverse events $(n=20)$ and those without $(n=572)$, which revealed that hospital stays were longer in the prior group (5.2 vs 3.0 days, $p<0.0001)$. C: The rate of discharge to home was compared between the cases with intraoperative adverse events $(n=21)$ and those without $(n=572)$, which revealed that the rate of discharge to home was lower in the prior group $(71.4 \%$ vs $88.5 \%, p=0.032)$.

and generalized seizure). Second, preoperative cognitive decline was assessed by medical practitioners who were members of the neurosurgery and anesthesia teams but was not formally assessed by psychiatric professionals. Third, as awake craniotomy was performed for various types of diseases, the database was not homogeneous, and numerous factors may have been confounding in the analyses. Although the database was constructed on a prospective basis, the charts were reviewed retrospectively, and thus not all of the clinical information could be collected, leaving the possibility of missing data, such as other important clinical/imaging factors that may have influenced the intraoperative adverse events. Last, as the study was unique in that all of the patients were operated on by a single surgeon and heterogeneous pathologies were included, the results may not be applicable to all institutions. However, the standard of care along with the surgical procedure and patient management were considered to be universal and similar to the practices of most tumor surgeons, so that the lessons learned in this study will inform daily neurosurgical decision-making.

\section{Conclusions}

The results of this study suggest that preoperative performance status is one of the most significant factors that 
affects whether patients can tolerate awake craniotomy for intracranial lesions. This finding again indicates that careful patient selection is key for the success of awake craniotomy. Based on this study, a recommended strategy that can be implemented to improve the approach to awake craniotomies is to make a list of criteria for preoperative indicators of risk for intraoperative adverse events to better anticipate additional patient postoperative care needs and thus reduce readmission rates and adverse outcomes.

\section{Acknowledgments}

We acknowledge all of the courageous patients who undergo awake (or asleep) craniotomy.

\section{References}

1. Dziedzic T, Bernstein M. Awake craniotomy for brain tumor: indications, technique and benefits. Expert Rev Neurother. 2014;14(12):1405-1415.

2. Purzner T, Purzner J, Massicotte EM, Bernstein M. Outpatient brain tumor surgery and spinal decompression: a prospective study of 1003 patients. Neurosurgery. 2011;69(1):119-127.

3. Duffau H. Stimulation mapping of white matter tracts to study brain functional connectivity. Nat Rev Neurol. 2015;11(5):255-265.

4. Hervey-Jumper SL, Li J, Lau D, et al. Awake craniotomy to maximize glioma resection: methods and technical nuances over a 27-year period. J Neurosurg. 2015;123(2):325-339.

5. Bernstein M. Subcortical stimulation mapping. J Neurosurg. 2004;100(3):365-366.

6. Mandonnet E, Duffau H. Mapping the brain for primary brain tumor surgery. In: Gunel JM, Piepmeier JM, Baehring JM, eds. Malignant Brain Tumors. Springer; 2017:63-79.

7. De Witt Hamer PC, Robles SG, Zwinderman AH, et al. Impact of intraoperative stimulation brain mapping on glioma surgery outcome: a meta-analysis. J Clin Oncol. 2012;30(20):2559-2565.

8. Marigil M, Bernstein M. Outpatient neurosurgery in neurooncology. Neurosurg Focus. 2018;44(6):E19.

9. Boulton M, Bernstein M. Outpatient brain tumor surgery: innovation in surgical neurooncology. J Neurosurg. 2008;108(4):649-654

10. Carrabba G, Venkatraghavan L, Bernstein M. Day surgery awake craniotomy for removing brain tumours: technical note describing a simple protocol. Minim Invasive Neurosurg. 2008;51(4):208-210.

11. Blanshard HJ, Chung F, Manninen PH, et al. Awake craniotomy for removal of intracranial tumor: considerations for early discharge. Anesth Analg. 2001;92(1):89-94.

12. Bernstein M. Outpatient craniotomy for brain tumor: a pilot feasibility study in 46 patients. Can J Neurol Sci. 2001;28(2):120-124.
13. Khu KJ, Doglietto F, Radovanovic I, et al. Patients' perceptions of awake and outpatient craniotomy for brain tumor: a qualitative study. J Neurosurg. 2010;112(5):1056-1060.

14. Conte V, Baratta P, Tomaselli P, et al. Awake neurosurgery: an update. Minerva Anestesiol. 2008;74(6):289-292.

15. Nobusawa S, Watanabe T, Kleihues P, Ohgaki H. IDH1 mutations as molecular signature and predictive factor of secondary glioblastomas. Clin Cancer Res. 2009;15(19):6002-6007.

16. Pasquet A. Combined regional and general anesthesia for craniotomy and cortical exploration. II. Anesthetic considerations. Curr Res Anesth Anal. 1954;33(3):156-164.

17. Serletis D, Bernstein M. Prospective study of awake craniotomy used routinely and nonselectively for supratentorial tumors. J Neurosurg. 2007;107(1):1-6.

18. Scuplak SM, Smith M, Harkness WF. Air embolism during awake craniotomy. Anaesthesia. 1995;50(4):338-340.

19. Balki M, Manninen PH, McGuire GP, et al. Venous air embolism during awake craniotomy in a supine patient. Can J Anaesth . 2003;50(8):835-838.

20. Kerrigan S, Erridge S, Liaquat I, et al. Mental incapacity in patients undergoing neuro-oncologic treatment: a crosssectional study. Neurology. 2014;83(6):537-541.

21. Bernstein M. Neuro-oncology: under-recognized mental incapacity in brain tumour patients. Nat Rev Neurol. 2014;10(9):487-488.

\section{Disclosures}

The authors report no conflict of interest concerning the materials or methods used in this study or the findings specified in this paper.

\section{Author Contributions}

Conception and design: Takami. Acquisition of data: Takami. Analysis and interpretation of data: Takami. Drafting the article: Takami. Critically revising the article: all authors. Reviewed submitted version of manuscript: all authors. Approved the final version of the manuscript on behalf of all authors: Takami. Administrative/technical/material support: Bernstein. Study supervision: Bernstein.

\section{Supplemental Information Online-Only Content}

Supplemental material is available with the online version of the article.

Supplementary Table 1. https://thejns.org/doi/suppl/10.3171/ 2020.4.JNS20378.

\section{Correspondence}

Mark Bernstein: Toronto Western Hospital, Toronto, ON, Canada. mark.bernstein@uhn.ca. 